OPEN ACCESS

Edited by:

Gillberto Ka Kit Leung,

The University of Hong Kong,

Hong Kong, SAR China

Reviewed by:

Sabino Luzzi,

University of Pavia, Italy

Songshan Feng,

Central South University, China

*Correspondence:

Weiduan Zhuang

zhuangweiduan@126.com

Specialty section:

This article was submitted to

Neurosurgery,

a section of the journal

Frontiers in Surgery

Received: 20 May 2021 Accepted: 17 September 2021

Published: 15 October 2021

Citation:

Liu S, Liu X and Zhuang W (2021)

Prognostic Factors Associated With

Survival in Patients With Diffuse

Astrocytoma. Front. Surg. 8:712350.

doi: 10.3389/fsurg.2021.712350

\section{Prognostic Factors Associated With Survival in Patients With Diffuse Astrocytoma}

\author{
Shuo Liu, Xiaoqiang Liu and Weiduan Zhuang* \\ Neurology Department, The First Affiliated Hospital of Shantou University Medical College, Shantou, China
}

Background: Diffuse astrocytoma (DA) is a rare disease with inadequately understood epidemiological characteristics and prognosis. Identification of the factors associated with the survival in DA patients is therefore necessary. In this study, we aim to investigate the clinicopathological characteristics of DA to delineate factors influencing the survival of DA.

Methods: A population-based cohort study was conducted, utilizing prospectively extracted data from the Surveillance, Epidemiology and End Results (SEER) database. Patients with histological diagnosis of DA in the SEER database from 1973 to 2017 were included.

Results: A total of 799 participants with DA were included, consisting of 95.9\% fibrillary astrocytoma and $4.1 \%$ protoplasmic variants. The average age of participants was 41.9 years, with $57.2 \%$ being male. The majority of the population was white (87.5\%). More than half (53.9\%) of the patients were married. DA arose mostly in the cerebrum (63.8\%). Around $71.6 \%$ of the population had received surgical treatment. The overall 1-, 3-, 5-, and 10-year survival rate were 73.7, 55.2, 49.4, and 37.6\%, respectively. Kaplan-Meier analysis showed that age at diagnosis, marital status, primary tumor site, tumor size, and surgery was possibly associated with cancer-specific survival (CSS) $(p<0.05)$. Multivariate Cox proportional hazard analysis indicated that surgery was a protective factor whereas older age, larger tumor size, and tumor in the brainstem were harmful factors for patients with DA. Moreover, a nomogram predicting 5- and 10-year survival probability for DA was developed.

Conclusions: Age, primary tumor site, tumor size, and surgery were associated with the survival of patients with DA.

Keywords: diffuse astrocytoma, epidemiology, prognosis, SEER database, nomogram

\section{INTRODUCTION}

Astrocytomas account for about $80 \%$ of adult gliomas. They are the most common gliomas in the fourth through the sixth decades of life. On the basis of histological features, astrocytomas are stratified into pilocytic astrocytoma (grade I), diffuse astrocytoma (DA) (grade II), anaplastic astrocytoma (grade III), and glioblastoma (grade IV) by the World Health Organization (WHO) (1). DA is an infiltrating, hypercellular tumor composed of atypical cells that show astrocytic differentiation and mildly increased mitotic activity (2). It comprises $10-15 \%$ of all astrocytic brain 
tumors (3). Although DA is defined as a WHO grade II tumor, it regularly undergoes a malignant transformation into anaplastic astrocytoma and glioblastoma, eventually resulting in death (4). DA consists of fibrillary astrocytoma, protoplasmic astrocytoma, and gemistocytic astrocytoma. Due to the rapid transformation into a higher grade (5), gemistocytic astrocytoma is considered a variant of anaplastic astrocytoma by some experts (6).

Clinical symptoms of DA vary depending on the location of the tumor. Seizures, headaches, and focal neurologic deficits are the most frequent presenting symptoms. In comparison with other WHO grade II tumors, DA has a relatively worse prognosis (7). On the basis of the United States national cancer registries (1995-2009), the 5-year overall survival (OS) rate of DA is $47.1 \%$ (8). In earlier studies, age is proved as a prognostic factor for survival in DA $(9,10)$. However, studies about clinicopathological characteristics of DA are scarce in the literature at present. Factors influencing the prognosis of DA are also unclear.

Therefore, we conducted an analysis of DA through the population-based SEER database. In this study, we aim to investigate the clinicopathological characteristics of DA to delineate factors influencing the survival of DA.

\section{METHODS}

\section{Patient Population}

The SEER program is a cancer registry that prospectively collects information of the patients, including clinicopathological characteristics of cancer and survival. It is supported by the National Cancer Institute. Our study was designed as a population-based retrospective study. We used the latest release data of the SEER database, which constituted of patients documented from the years 1973-2017. According to the International Classification of Diseases for Oncology, Third Edition (ICD-O-3), patients diagnosed with DA as their primary tumor were identified. The ICD-O-3 morphology codes were 9,410 (protoplasmic astrocytoma) and 9,420 (fibrillary astrocytoma). Demographic features of these patients and clinicopathological characteristics of DA were collected.

\section{Definition of Variables}

The age of the patient at diagnosis, race, sex, marital status, primary tumor site, histological type, tumor size, surgical treatment, survival duration in months, and survival status were collected in this study. Patients with unclear information on any of the collected variables were excluded. The race was grouped into three categories: white, black, and others (American Indian/Alaska Native, Asian, or Pacific Islander). Similarly, marital status at diagnosis was classified as single, married, and others (separated/divorced/widowed) (Sep/Div/Wid). The primary tumor site was recategorized into five distinct categories including cerebrum (C71.1/C71.2/C71.3/C71.4/C71.5), cerebellum (C71.6), brainstem (C71.7), spinal cord (C72.0), and others. The primary outcome was cancer-specific survival (CSS).

\section{Statistical Analysis}

Cancer-specific survival stratified by each factor was delineated by a Kaplan-Meier curve. The connection between
TABLE 1 | Characteristics of patients with diffuse astrocytoma.

\begin{tabular}{llc}
\hline Characteristics & & Number \\
\hline Number & & 799 \\
Age (year) \pm SD & Male & $41.9 \pm 20.9$ \\
Sex & Female & $457(57.2 \%)$ \\
Race & White & $342(42.8 \%)$ \\
& Black & $699(87.5 \%)$ \\
Marital status & Others & $56(7.0 \%)$ \\
& Single & $44(5.5 \%)$ \\
Primary site & Married & $271(33.9 \%)$ \\
& Others & $431(53.9 \%)$ \\
& Brainstem & $97(12.2 \%)$ \\
Cerebrum & $28(3.5 \%)$ \\
Histology & Cerebellum & $510(63.8 \%)$ \\
Tumor size (mm) & Spinal cord & $38(4.8 \%)$ \\
Surgery & Others & $14(1.8 \%)$ \\
& Protoplasmic & $209(26.1 \%)$ \\
Status & Fibrillary & $33(4.1 \%)$ \\
& $41.1 \pm 19.8$ & $766(95.9 \%)$ \\
& Yes & \\
& No & $572(71.6 \%)$ \\
& Alive & $227(28.4 \%)$ \\
& Dead & $326(40.8 \%)$ \\
& & $473(59.2 \%)$ \\
\hline
\end{tabular}

clinicopathological factors and CSS was analyzed using Cox proportional hazards model. Statistically significant variables in univariate Cox analysis were further included in multivariate Cox analysis. For each patient, significant prognostic factors were further put into the nomogram calculator to get a predicted survival rate at 5- and 10 years. The $\mathrm{C}$-index and receiver operating characteristic (ROC) curve were utilized to evaluate the accuracy of the nomogram. The C-index and area under the curve (AUC) ranged from 0.5 to 1 . A Higher C-index value or AUC indicated a better prognostic model. A two-tailed $P \leq 0.05$ was determined as statistically significant. R software (version 3.5.0) was utilized to perform the statistical analysis.

\section{RESULTS}

\section{Incidence}

A total of 799 patients with DA(mean age 41.9 years) were included (Table 1), consisting of 766 fibrillary astrocytoma (95.9\%) and 33 protoplasmic variants (4.1\%). Among this population, 457 were male (57.2\%). The majority of the population was white $(87.5 \%)$. Most subjects were married (53.9\%), whereas $33.9 \%$ were single. Of these patients, DA arose mostly in the cerebrum (63.8\%), while to a lesser extent in the cerebellum (4.8\%), brainstem (3.5\%), and spinal cord (1.8\%). With respect to tumor characteristics, the mean tumor size was $41.1 \mathrm{~mm}$. Around $71.6 \%$ of the population had surgery to decrease the tumor burden. Among all the cases, DA accounted for 436 deaths (54.6\%). 

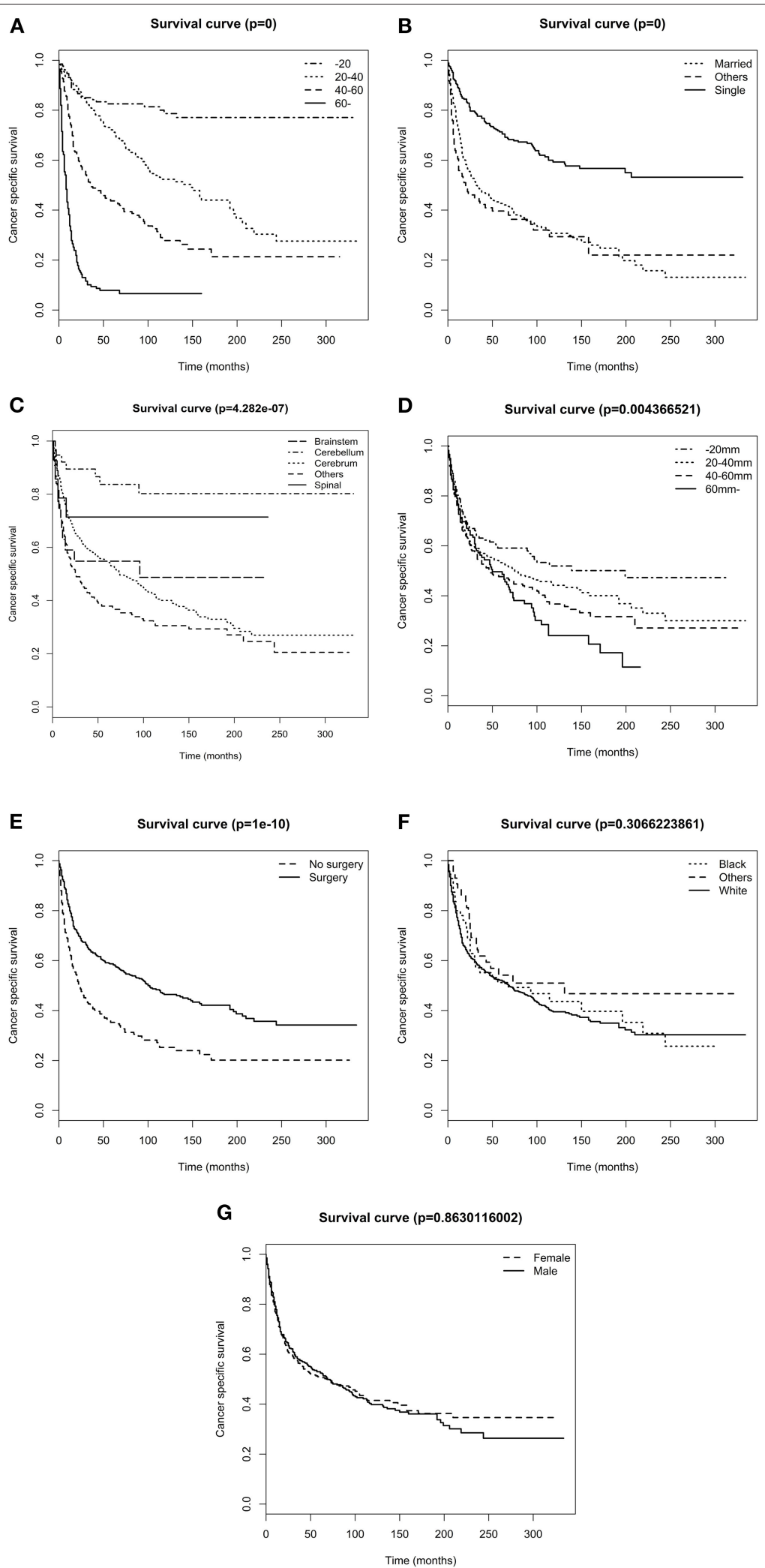

FIGURE 1 | Survival analysis of patients stratified by risk factors. (A) age (B) marital status, (C) primary tumor site, (D) tumor size, (E) surgery, (F) race, and (G) sex. 
TABLE 2 | Univariate and multivariate Cox proportional hazard analyses of clinical characteristics for cancer-specific survival rates.

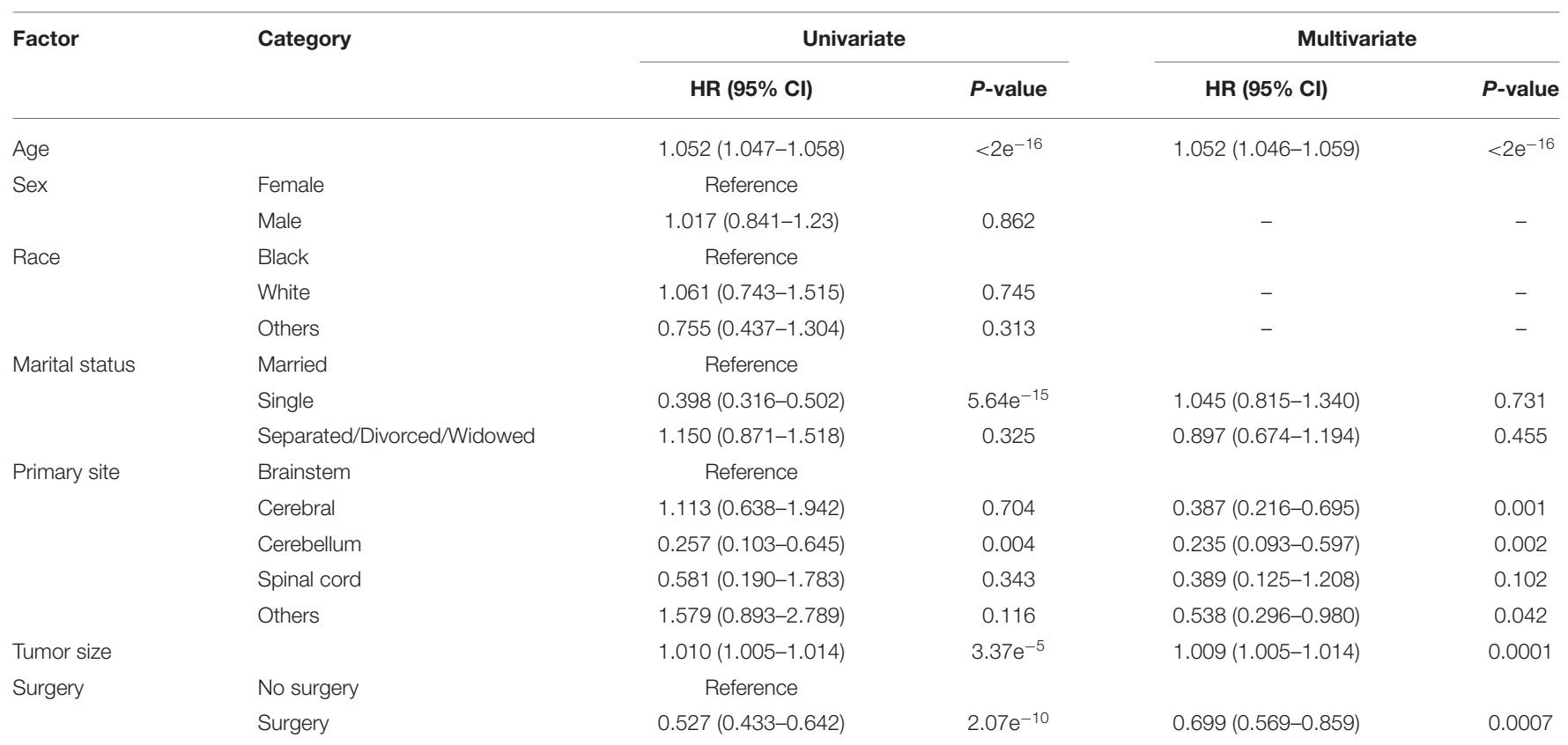

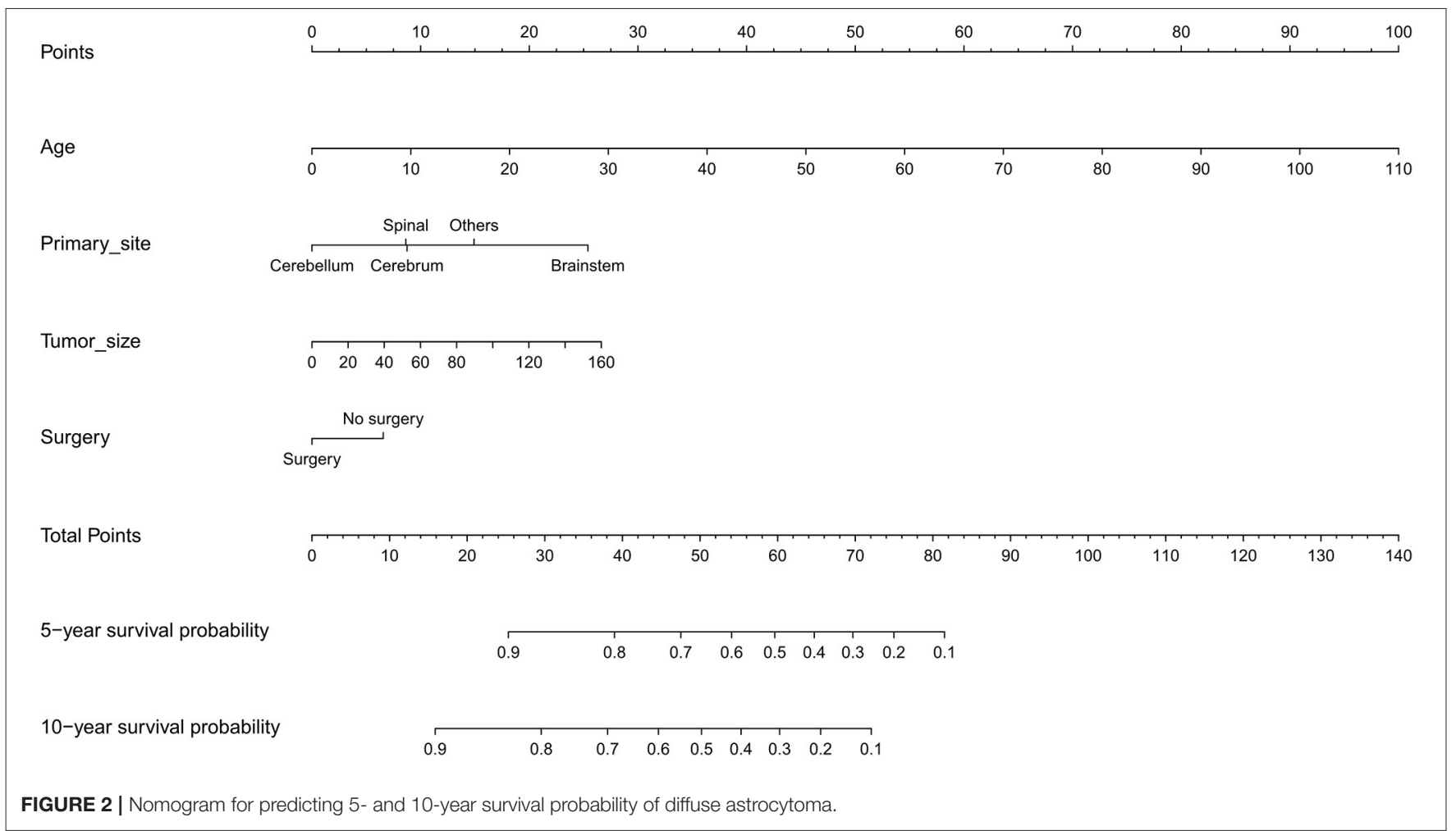

\section{Survival}

The overall 1-, 3-,5-, and 10-year survival rates were 73.7, $55.2,49.4$, and $37.6 \%$, respectively. The cancer specific 1-, 3-, 5-, and 10-year survival rates were 75.2, 57.3, 51.8, and $40.5 \%$, respectively. The Kaplan-Meier analysis showed that age at diagnosis (Figure 1A), marital status (Figure 1B), primary tumor site, (Figure 1C), tumor size (Figure 1D), and surgery (Figure 1E) were possibly associated with CSS, whereas race (Figure 1F) and sex (Figure 1G) were not associated with CSS. All included variables were admitted into the univariate Cox analysis. As shown in Table 2, the results showed that age, marital status, primary tumor site, tumor size, and surgery each 


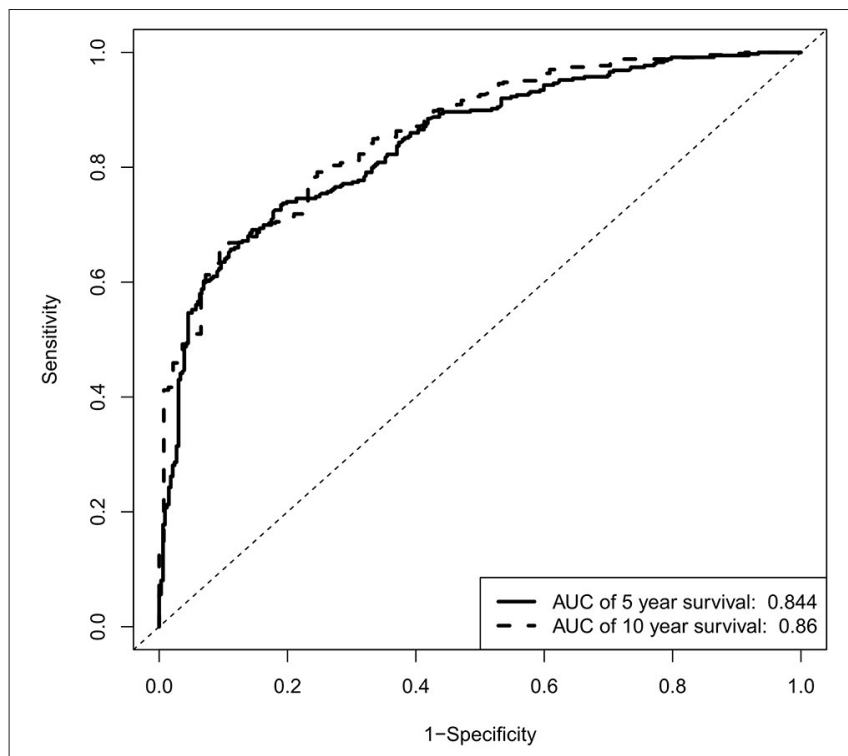

FIGURE 3 | ROC curves for validating nomogram model.

had statistically significant association with CSS $(p<0.05)$. No significant difference was noticed across sex and race. Significant variables in the univariate Cox analysis were further included in the multivariate Cox analysis, and the result demonstrated that age, primary tumor site, tumor size, and surgery were independent prognostic factors for DA. Surgery was a protective factor whereas older age, larger tumor size, and tumor in the brainstem were harmful factors for patients with DA.

\section{Nomogram}

Based on the independent predictors from the multivariate Cox proportional hazards analysis, a nomogram predicting 5- and 10-year CSS for each of the DA predictors was constructed (Figure 2). This nomogram showed the probability of involvement of each predictor, and higher points correlated with lower survival probability. It also revealed that age contributed most to the prognosis, followed by tumor size, primary site, and surgery. By adding the scores of each predictor, the CSS probability of each patient with DA can be calculated. An interesting observation was that DA in the brainstem led to the lowest survival probability compared with other sites. Likewise, older patients and larger tumor sizes would appear to indicate a lower survival probability. The $\mathrm{C}$-index of the nomogram was 0.774. In the plotted ROC curves, the AUC was 0.844 and 0.860 at 5 - and 10 -years, indicating a moderate accuracy in our nomogram model (Figure 3). Moreover, an optimal agreement between actual observation and the nomogram prediction was seen in the calibration plot for the probability of survival at 5or 10-years (Figures $\mathbf{4 A , B}$ ).

\section{DISCUSSION}

Reports are scarce regarding the detailed clinicopathological characteristics of DA. Most present studies of DA are small

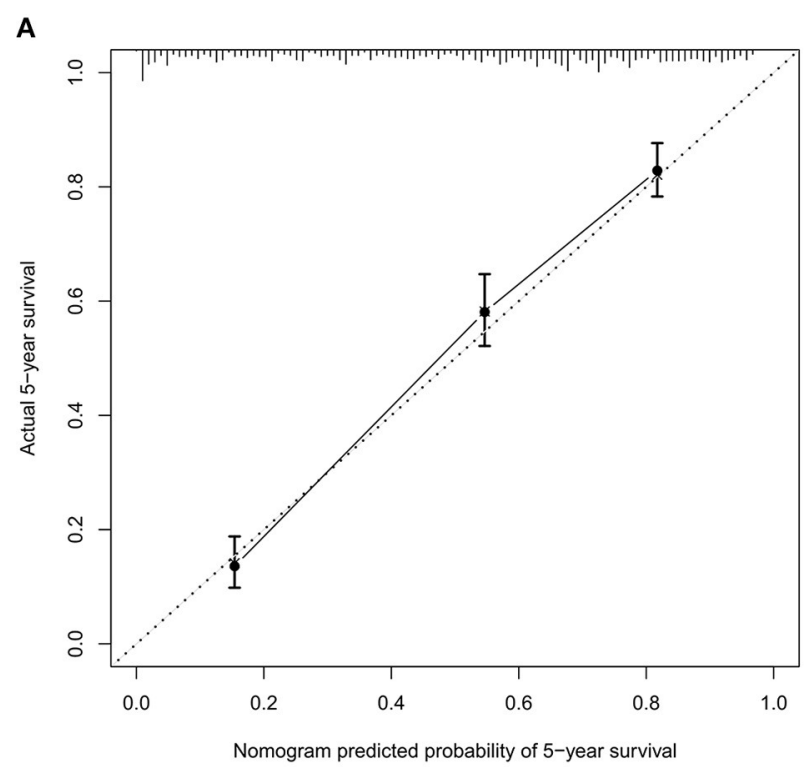

B

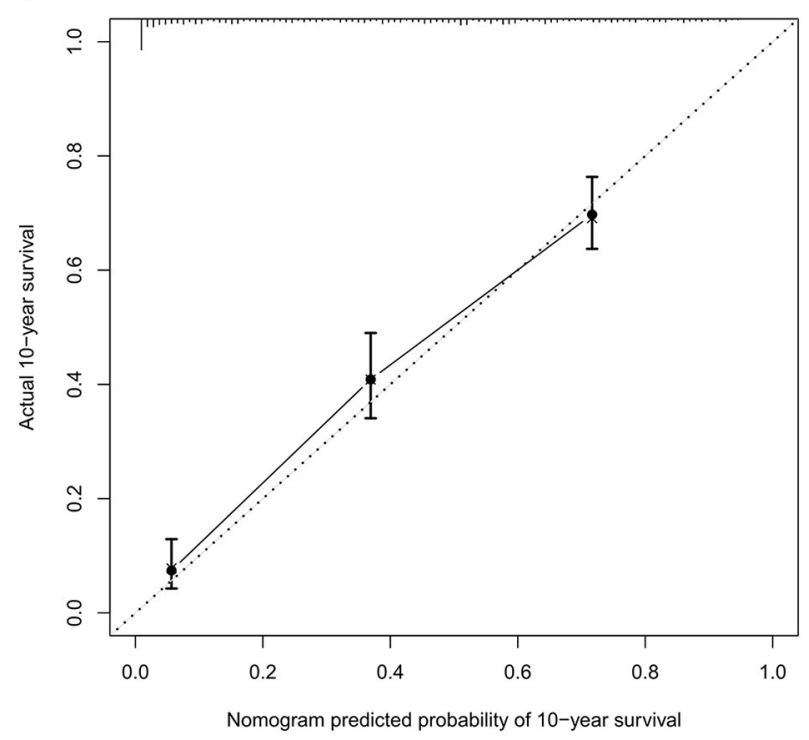

FIGURE 4 | The calibration curve for predicting patient survival at 5- (A) and 10-years (B). Nomogram predicted probability of cancer-specific survival (CSS) is plotted on the $x$-axis, actual CSS is plotted on the $y$-axis.

case series. Therefore, epidemiological characteristics and factors influencing the prognosis of DA remain unclear. To the best of our knowledge, our study is the largest one to depict clinicopathological characteristics of DA and describe factors influencing its survival.

Diffuse astrocytoma has been previously reported in younger people. In our study, the average age at diagnosis was 41.9 years. The literature suggested that the mean age at diagnosis of DA patients was 45.7 years, which was slightly older than our subjects (11). This disease mostly occurs in young people, which increases the economic and social burden for the entire family and society. 
Our data demonstrated that the white was predominately affected and over half of the patients were married. We also found that DA most commonly arose in the cerebrum. The previous study had reported that DA was more prevalent among men (51.7:48.3 male: female) (12) and our male:female (57.2:42.8) ratio was slightly higher when compared with literature. In literature, protoplasmic astrocytoma was a rare variant and fibrillary type was the most common, representing more than $85 \%$ of all DA (13). Our data showed that only $4.1 \%$ were protoplasmic astrocytoma whereas $95.9 \%$ were fibrillary astrocytoma. The previous study had reported that the 5- and 10-year survival rates for DA were 48 and 36\%, respectively (14). In our study, $49.4 \%$ lived more than 5 years whereas $37.6 \%$ lived more than 10 years.

Among the clinical variables, age was identified as a significant prognostic factor for the survival of DA in our study. Older patients had a much poorer prognosis. This result was in line with the literature $(10,15-18)$. A previous study had put forward the definite association between age and survival of DA, with a worse prognosis in older patients (9). Moreover, age values of 47 and 63 years were calculated as two optimal cutoff values to distinguish good, moderate, and poor survival.

Surprisingly, we observed that tumor size affected the survival of DA. Similarly, the tumor size value of 25 and $44 \mathrm{~mm}$ were two optimal cutoff values to distinguish good, moderate, and poor survival. We were also the first to report the effect of primary tumor site on the survival of DA. DA in the brainstem had the worst survival rate compared with those in other sites. A possible interpretation is that the brainstem plays an important role in the regulation of cardiac and respiratory function. Tumor in the brainstem is more likely to affect heart rate and breathing than tumors in other sites and it causes a lower survival rate.

Evidence concerning the effect of surgery in DA is rare in literature. Our study revealed that surgery was associated with better survival in DA. However, the surgical types of most included cases were not specified in the database. Therefore, we were unable to further analyze the association between surgical types and survival. Previous research demonstrated that extent of tumor resection was not correlated with patient survival in DA (3). Due to the scarce evidence in DA, we used evidence in low grade glioma (LGG) for reference. In the last few decades, the value of surgery in LGG was controversial. However, recent viewpoints have suggested that biopsy is considered harmful, whereas extensive resection is correlated with a more favorable prognosis $(2,19)$. Some new concepts also have put forward a new standpoint that supratotal resection is a protective factor relating to the management of LGG $(20,21)$. However, these conclusions come from studies of LGG, which contain not only DA but also oligodendroglioma and oligoastrocytoma. More studies are needed to prove the value of surgery in DA.

A nomogram is an essential tool of modern medical decision-making (22). It is a graphical demonstration of a statistical prediction model generating survival probability of a specific outcome $(23,24)$. Doctors could easily figure out the prognosis for patients by using a nomogram of an efficient prognostic system. This could also assist in patient counseling and individualized treatment. Additionally, nomograms are specifically worthy for clinicians to solve complex diseases where no definite clinical guidelines exist. Therefore, we constructed a nomogram predicting CCS of DA based on a large population from the SEER database. The nomogram performed well in predicting survival probability, supported by the $\mathrm{C}$-indexes (0.774) and the ROC curves (AUC of 0.844 and 0.860 at 5and 10-years, respectively). Prediction of prognosis of patients by utilizing nomograms is straightforward. Firstly, each variable corresponds to relevant "points" in the nomogram by drawing a vertical line. Secondly, "total points" is obtained by summing up all the "points" of each variate. Finally, a vertical line from "total points" to the "survival probability" is drawn to get the corresponding survival probability. For example, consider a 22year-old (20 points) DA patient, with tumor location of the brainstem (28 points), tumor size of $20 \mathrm{~mm}$ (4 points), and received surgery ( 0 points). After using our nomogram, the "total points" of this patient are 52. The 5- and 10-year CCS probabilities of this patient are about 63 and $48 \%$, respectively.

Our study had several limitations. Firstly, DA is currently defined by both histologic and molecular characteristics, whereas the SEER database does not contain the relevant molecular information (25). However, due to the high degree of accordance (about $80 \%$ ) between the histologic diagnosis and the molecular diagnosis of glioma, this limitation may be alleviated $(26,27)$. Secondly, we lacked information about radiotherapy, which may play an important part in survival in DA patients. Ultimately, missing data and selection bias were inevitable because the study design was retrospective.

\section{CONCLUSIONS}

In conclusion, our study is the largest one to date to investigate the clinicopathological characteristics and survival for patients with DA. We found that age, primary tumor site, tumor size, and surgery were associated with the survival of patients with DA. These outcomes may contribute to future management in DA patients.

\section{DATA AVAILABILITY STATEMENT}

The datasets presented in this study can be found in online repositories. The names of the repository/repositories and accession number(s) can be found in the article/supplementary material.

\section{AUTHOR CONTRIBUTIONS}

WZ: conceptualization. SL: formal analysis, project administration, and software. SL, XL, and WZ: methodology. WZ: supervision. SL and XL: writing-original draft. SL and WZ: writing - review and editing. All authors contributed to the article and approved the submitted version. 


\section{REFERENCES}

1. Louis DN, Ohgaki H, Wiestler OD, Cavenee WK, Burger PC, Jouvet A, et al. The 2007 WHO classification of tumours of the central nervous system. Acta Neuropathol. (2007) 114:97-109. doi: 10.1007/s00401-007-0243-4

2. Bourne TD, Schiff D. Update on molecular findings, management and outcome in low-grade gliomas. Nat Rev Neurol. (2010) 6:695701. doi: 10.1038/nrneurol.2010.159

3. Ranjan M, Santosh V, Tandon A, Anandh B, Sampath S, Devi BI, et al. Factors predicting progression of low-grade diffusely infiltrating astrocytoma. Neurol India. (2011) 59:248-53. doi: 10.4103/0028-3886.79137

4. Narita Y. Current knowledge and treatment strategies for grade II gliomas. Neurol Med Chir. (2013) 53:429-37. doi: 10.2176/nmc.53.429

5. Watanabe K, Tachibana O, Yonekawa Y, Kleihues P, Ohgaki H. Role of gemistocytes in astrocytoma progression. Lab Invest. (1997) 76:277-84.

6. Krouwer HG, Davis RL, Silver P, Prados M. Gemistocytic astrocytomas: a reappraisal. J Neurosurg. (1991) 74:399-406. doi: 10.3171/jns.1991.74.3.0399

7. Narita Y. Chemotherapy of diffuse astrocytoma (WHO grade II) in adults. Prog Neurol Surg. (2018) 31:145-51. doi: 10.1159/000467375

8. Dolecek TA, Propp JM, Stroup NE, Kruchko C. CBTRUS statistical report: primary brain and central nervous system tumors diagnosed in the United States in 2005-2009. Neuro Oncol. (2012) 14(Suppl. 5):v149. doi: 10.1093/neuonc/nos 218

9. Pignatti F, van den Bent M, Curran D, Debruyne C, Sylvester R, Therasse P, et al. Prognostic factors for survival in adult patients with cerebral low-grade glioma. J Clin Oncol. (2002) 20:2076-84. doi: 10.1200/JCO.2002.08.121

10. Lote K, Egeland T, Hager B, Stenwig B, Skullerud K, Berg-Johnsen J, et al. Survival, prognostic factors, and therapeutic efficacy in low-grade glioma: a retrospective study in 379 patients. J Clin Oncol. (1997) 15:312940. doi: 10.1200/JCO.1997.15.9.3129

11. Wegman-Ostrosky T, Reynoso-Noveron N, Mejia-Perez SI, Sanchez-Correa TE, Alvarez-Gomez RM, Vidal-Millan S, et al. Clinical prognostic factors in adults with astrocytoma: historic cohort. Clin Neurol Neurosurg. (2016) 146:116-22. doi: 10.1016/j.clineuro.2016.05.002

12. Babu R, Bagley JH, Park JG, Friedman AH, Adamson C. Lowgrade astrocytomas: the prognostic value of fibrillary, gemistocytic, and protoplasmic tumor histology. J Neurosurg. (2013) 119:43441. doi: 10.3171/2013.4.JNS122329

13. Ajlan A, Recht L. Supratentorial low-grade diffuse astrocytoma: medical management. Semin Oncol. (2014) 41:44657. doi: 10.1053/j.seminoncol.2014.06.013

14. Davis FS. Epidemiology of brain tumors. Expert Rev Anticancer Ther. (2007) 7(12 Suppl):S3-6. doi: 10.1586/14737140.7.12s.S3

15. Leighton C, Fisher B, Bauman G, Depiero S, Stitt L, MacDonald D, et al. Supratentorial low-grade glioma in adults: an analysis of prognostic factors and timing of radiation. J Clin Oncol. (1997) 15:1294301. doi: 10.1200/JCO.1997.15.4.1294

16. van Veelen ML, Avezaat CJ, Kros JM, van Putten W, Vecht C. Supratentorial low grade astrocytoma: prognostic factors, dedifferentiation, and the issue of early versus late surgery. J Neurol Neurosurg Psychiatry. (1998) 64:5817. doi: 10.1136/jnnp.64.5.581

17. Laws ER Jr. Neurosurgical management of low-grade astrocytoma of the cerebral hemisphere. J Neurosurg. (1985) 63:819. doi: 10.3171/jns.1985.63.5.0819a
18. Nicolato A, Gerosa MA, Fina P, Iuzzolino P, Giorgiutti F, Bricolo A. Prognostic factors in low-grade supratentorial astrocytomas: a unimultivariate statistical analysis in 76 surgically treated adult patients. Surg Neurol. (1995) 44:208-21; discussion 21-3. doi: 10.1016/0090-3019(95) 00184-0

19. Duffau H, Taillandier L. New concepts in the management of diffuse lowgrade glioma: proposal of a multistage and individualized therapeutic approach. Neuro Oncol. (2015) 17:332-42. doi: 10.1093/neuonc/ nou 153

20. Yordanova YN, Moritz-Gasser S, Duffau H. Awake surgery for WHO Grade II gliomas within "noneloquent" areas in the left dominant hemisphere: toward a "supratotal" resection. Clinical article. J Neurosurg. (2011) 115:2329. doi: 10.3171/2011.3.JNS101333

21. Duffau H. Long-term outcomes after supratotal resection of diffuse low-grade gliomas: a consecutive series with 11-year follow-up. Acta Neurochir. (2016) 158:51-8. doi: 10.1007/s00701-015-2621-3

22. Balachandran VP, Gonen M, Smith JJ, DeMatteo RP. Nomograms in oncology: more than meets the eye. Lancet Oncol. (2015) 16:e17380. doi: 10.1016/S1470-2045(14)71116-7

23. Kattan MW, Leung DH, Brennan MF. Postoperative nomogram for 12-year sarcoma-specific death. J Clin Oncol. (2002) 20:7916. doi: 10.1200/JCO.2002.20.3.791

24. Bochner BH, Kattan MW, Vora KC. Postoperative nomogram predicting risk of recurrence after radical cystectomy for bladder cancer. J Clin Oncol. (2006) 24:3967-72. doi: 10.1200/JCO.2005.05.3884

25. Louis DN, Perry A, Reifenberger G, von Deimling A, Figarella-Branger D, Cavenee WK, et al. The 2016 World Health Organization classification of tumors of the central nervous system: a summary. Acta Neuropathol. (2016) 131:803-20. doi: 10.1007/s00401-016-1545-1

26. Boots-Sprenger SH, Sijben A, Rijntjes J, Tops BB, Idema AJ, Rivera AL, et al. Significance of complete 1p/19q co-deletion, IDH1 mutation and MGMT promoter methylation in gliomas: use with caution. Mod Pathol. (2013) 26:922-9. doi: 10.1038/modpathol.2012.166

27. Yan H, Parsons DW, Jin G, McLendon R, Rasheed BA, Yuan W, et al. IDH1 and IDH2 mutations in gliomas. N Engl J Med. (2009) 360:76573. doi: 10.1056/NEJMoa0808710

Conflict of Interest: The authors declare that the research was conducted in the absence of any commercial or financial relationships that could be construed as a potential conflict of interest.

Publisher's Note: All claims expressed in this article are solely those of the authors and do not necessarily represent those of their affiliated organizations, or those of the publisher, the editors and the reviewers. Any product that may be evaluated in this article, or claim that may be made by its manufacturer, is not guaranteed or endorsed by the publisher.

Copyright (c) 2021 Liu, Liu and Zhuang. This is an open-access article distributed under the terms of the Creative Commons Attribution License (CC BY). The use, distribution or reproduction in other forums is permitted, provided the original author(s) and the copyright owner(s) are credited and that the original publication in this journal is cited, in accordance with accepted academic practice. No use, distribution or reproduction is permitted which does not comply with these terms. 